ARTÍCULO ORIGINAL

\title{
LA EDUCACIÓN Y SU INCIDENCIA EN EL CRECIMIENTO ECONÓMICO BAJO UNA ECONOMÍA GLOBAL
}

\section{EDUCATION AND ITS INCIDENCE IN ECONOMIC GROWTH UNDER A GLOBAL ECONOMY}

\author{
Nicko Alberto Gomero Gonzales \\ Doctor en Ciencias Económicas - Docente Principal de la Facultad de Ciencias Contables - Universidad Nacional Mayor de San \\ Marcos, Lima, Perú - Email: ngomerog@unmsm.edu.pe (Autor Corresponsal)
}

[Recibido: 15/10/2016 Aceptado: 16/01/2017]

\begin{abstract}
RESUMEN
La inversión es un componente básico para generar crecimiento económico. Debiendo orientarse al establecimiento de políticas públicas que potencien el conocimiento, el desarrollo de la ciencia, la tecnología y la investigación, que son factores importantes para alcanzar estándares de calidad en el escenario social y económico. El presente estudio es de tipo descriptivo, correlacional y causal, en donde se evidencia la relación entre la inversión en educación e investigación y desarrollo (I\&D) en el proceso del crecimiento económico bajo una economía globalizada. Se evidencia que los países en vías de desarrollo orientan sus inversiones a actividades extractivas, quedando ancladas a los vaivenes del precio de los commodities. Además, se dejan fuera de las agendas gubernamentales la creación de políticas educativas que son el pilar básico en países con economías más sólidas que la nuestra, tal y como ha sido demostrado en esta investigación.
\end{abstract}

\section{PALABRAS CLAVE}

Crecimiento económico, investigación, innovación, política educativa, educación.

\begin{abstract}
Investment is a basic component to generate economic growth. It should be oriented to the establishment of public policies that enhance knowledge, the development of science, technology and research, which are important factors for achieving quality standards in the social and economic scenario. The present study is descriptive, correlational and causal. The relationship between investment in education and research and development $(R \& D)$ in the process of economic growth under a globalized economy is evidenced. The developing countries orient their investments to extractive activities, being anchored to the fluctuations of the price of commodities. Furthermore, they leave outside the governmental agendas the creation of educational policies that are the basic pillar in countries with more solid economies than ours, as it has been demonstrated in this research.
\end{abstract}

\section{KEYWORDS}

Economic growth, research, innovation, educational policy, education.

Como Citar: Gomero, N. (2017). La educación y su incidencia en el crecimiento económico bajo en una economía global. Quipukamayoc, 25(47), 45 - 54. doi: http://dx.doi.org/10.15381/quipu.v25i47.13802

\section{INTRODUCCIÓN}

Para obtener un crecimiento económico sólido y sostenible es importante que se aperturen espacios para las inversiones sectoriales, especialmente aquellas que están dirigidas a generar valor agregado y con la máxima responsabilidad social. Los estudios econométricos han demostrado que los efectos multiplicadores de este componente en el PBI son altamente significativos, a la vez de contar con alto impacto en el mercado laboral. Pero, como era de esperar, las políticas gubernamentales han apuntado siempre a actividades tradicionales, como las extractivas o la de infraestructura, dejando espacios marginales a otros tipos de inversiones que son básicas para dar sostenibilidad al crecimiento de la economía en un largo plazo, el cual está basado en el capital humano. La inversión en la industria del cono- cimiento que está orientada a fortalecer las capacidades o competencias de las personas en el sector educación, conjuntamente con la investigación e innovación, son los pilares básicos para que una economía logre resultados importantes en escenarios globalizados.

Antes se hablaba del BRICS como ejes de la economía mundial, ahora esta cartera de países ha sido remplazada por las TICK (Taiwán, India, China, 
Corea del Sur) economías emergentes que han logrado mejores posicionamientos en el ránking de Competitividad Global (2015-2016), gracias en parte a la exitosa política educativa, que se plasma en la inversión en el capital humano.

Ni que decir de Suiza, Japón, Alemania, Finlandia, cuyo logro como los países más competitivos del mundo se debe al fortalecimiento del pilar educativo. Si apreciamos los resultados económicos y sociales de ciertos países en proceso de desarrollo, como es el caso de Perú, estos son poco alentadores, es más, muestran debilidades estructurales, el cual es simplemente un derivado de la débil incidencia de la I\&D en el campo económico.

En el presente artículo se demostrará la importancia de la educación y en términos más amplios de la Investigación y Desarrollo (I\&D) en el proceso de crecimiento en un largo plazo, que es el camino crítico para lograr mejores niveles de bienestar para la población.

Existe un conjunto de teorías sobre el crecimiento económico en donde se pone de manifiesto la importancia del acervo tecnológico, siendo este un derivado de la educación. En las teorías clásicas y neoclásicas de corte económico se fundamentan conceptos que explican que una economía no puede generar fortalezas de sostenibilidad a largo plazo si es que las fuerzas de la inversión no aterrizan en componentes cuyos efectos multiplicadores se aprecien en un largo plazo, estas son por cierto, las que están ligadas a la investigación y la educación, pilares básicos para lograr mejoras del escenarios económico y social.

Rodríguez (2005) señala que los estudios de Solow y sus contemporá- neos demostraron cuantitativamente la importancia de la acumulación de capital y el progreso tecnológico en la producción de largo plazo, pero sobre todo vieron a la tecnología, la calificación de la mano de obra y las innovaciones como principales motores del crecimiento económico.

¿Por qué es relevante el aporte de Solow? El grado de importancia se fundamenta en el hecho que los factores tecnológicos y capital humano al constituirse, según el modelo planteado, forman componentes críticos para afianzar el proceso de crecimiento económico.

Larrain y Sachs (2002) explican la teoría de Solow planteando la siguiente ecuación matemática:

\section{Donde}

$$
\mathrm{Q}=\mathrm{T} \times \mathrm{F}(\mathrm{K}, \mathrm{L})
$$

\section{K: Representa al acervo capital \\ L: insumo laboral \\ T: estado de la tecnología}

Como lo señala los autores antes referido el crecimiento económico proviene del aumento del capital, del trabajo o del progreso tecnológico. Larrain y Sachs (2002, p. 105) precisan que: "Solow demostró como el número de productos puede distribuirse entre estos tres factores".

Estableciendo ciertos supuestos sobre la función de producción y asumiendo una economía de mercado competitivo. La ecuación que subyace de lo señalado según la teoría de Solow es la siguiente:

$$
\mathrm{dQ} / \mathrm{q}=\mathrm{dT} / \mathrm{T}+\mathrm{sl}(\mathrm{dL} / \mathrm{L})+\mathrm{sk}(\mathrm{dK} / \mathrm{K})
$$

De acuerdo con la ecuación planteada el cambio de la producción depende del cambio tecnológico, del grado de participación de la fuerza laboral y del factor capital. Todos estos componentes como se aprecia en el modelo matemático, esta correlacionada del monto en que se pueda invertir en cada factor considerado.

Cabe resaltar que los factores señalados por Larrain y Sachs (2002) impactan en el PBI, pero cabe recordar que este indicador refleja el crecimiento económico, pero deja de lado las asimetrías sociales que pudiera darse en una sociedad.

Hay nuevas teorías de crecimiento económico después de Solow que también explican la importancia del factor humano y del capital en el proceso de crecimiento económico, la diferencia radica que esta última es más refinada y descompone los factores dentro del modelo pero al operativizarla llegan a los mismos resultados, cabe destacar que en esta nueva teoría se da relevancia a la educación como elemento crítico para potenciar la productividad o rendimiento de los recursos humanos. Esta teoría fue trabajada por Denison Edward de la Institución Brookings, Griliches y Dale Rogerson de la Universidad de Harvard que con información para la economía norteamericana llegan a demostrar la sensibilidad del crecimiento a estos tipos de factores.

Larrain y Sach(2002) señalan: Denison llega a demostrar que la educación juega un papel importante como factor determinante del aumento del producto por trabajador. Esto demuestra la importancia de invertir en el capital humano. A través de su investigación realizada para la economía norteamericana cuyo período de evaluación fue de 53 años se llegó a demostrar que la profundidad del capital humano es decir el aumento de este factor por cada unidad de trabajador es responsable del 15\% del aumento del Producto Bruto Interno resultado también al que arribo Robert Solow. 
Dornbush, Fisher, Startz (2015) precisan que: "En los países industrializados el trabajo es menos importante que la destreza y habilidades de los trabajadores el acervo de la sociedad de dichas habilidades se acrecienta mediante inversiones en capital humano, por ejemplo: escolarización, capacitación laboral y otros medios de la misma manera que la inversión física incrementa el capital físico”.

Gerald Destinobles (2007) explica sobre el crecimiento endógeno, señalando que existen cuatro factores que explican este resultado, los cuales son:

a) El capital físico atribuye el crecimiento a la acumulación de capital (Romer, 1986, citado en Gerald, 2007).

b) El capital público de infraestructura

c) Investigación y desarrollo; que en los trabajos de Romer son considerados como una actividad que generan rendimientos crecientes y donde se pone en evidencia la correlación entre la investigación y el desarrollo para los países de la Organización para la Cooperación y el Desarrollo Económico (OCDE) (Coe y Helpman, 1993, citado en Gerald, 2007).

d) Capital Humano, definido como el stock de conocimientos que es valorizado económicamente e incorporado por los individuos (calificación, estado de salud, higiene...).

La idea de acumulación de capital siendo que el capital humano voluntario que corresponde a una acumulación de conocimientos (schooling) y la acumulación involuntaria (learning by doing). Al mejorar su nivel de educación y de formación cada persona aumenta el stock de capital de una nación y de allí contribuye al mejoramiento de la productividad de la economía nacional, es decir, la productividad privada del capital humano tiene un efecto externo positivo (Lucas, 1988, citado en Hanushek y Wößmann, 2010).

La relación de causalidad entre la educación y la economía también se aprecia en el informado preparado por la UNESCO (2015) en donde señala “El progreso educativo de los países, no es sino un componente del proceso de mejoramiento

de las condiciones de vida de las sociedades, es decir su desarrollo guarda con este una relación de influencia recíproca aunque se espera que una más extendida y mejor educación contribuya al progreso general, la misma ausencia de progreso constituye a su vez un obstáculo a la expansión de las oportunidades educativas."

Hay que precisar que la educación y todos los factores que subyacen de esta actividad estratégica no solo son utilizados como herramientas para generar mayores crecimientos económicos y a la vez darle sostenibilidad, sino que además es una de las rutas más importantes con que cuenta un gobierno para generar simetrías sociales en la población, es decir, la educación se convierte de esta forma en una herramienta para crear escenarios de igualdad de oportunidades.

\section{MATERIAL Y MÉTODOS}

El trabajo de investigación presentado es de tipo descriptivo, correlacional y causal, en donde se evidencia la relación entre la inversión en educación e investigación y desarrollo (I\&D) en el proceso de crecimiento económico bajo una economía globalizada. Para ello, se utilizó información obtenida de fuentes oficiales, como la UNESCO, Banco Mundial, MEF, que fueron debidamente pro- cesados, utilizando para ello herramientas estadísticas e informáticas.

\section{RESULTADOS}

Educación, Investigación y Desarrollo (I\&D) y su incidencia en el crecimiento económico

\section{Educación y la economía mundial}

La supremacía economía mundial, lo cual no implica que esto se refleja en el aspecto social, sigue concentrado en cuatro países, como son EE.UU, China, Japón, Alemania. Pero este resultado se da con mayor relevancia en los dos primeros países que, prácticamente tienen la hegemonía mundial. $\mathrm{Su}$ fortaleza productiva ha hecho que estos países generen riqueza que se ha transformado en mayores niveles de bienestar. Por cierto este último objetivo no es simétrico ya que persisten las desigualdades sociales y económicas, pero sí de hecho cuentan con mejores escenarios en comparación con aquellos países como los de LAC (Latinoamérica y el Caribe) que no han sabido explotar sus fortalezas competitivas y comparativas. Según el FMI, las dos economías líderes del mundo son responsables en promedio del 33\% del PBI mundial atribuyéndole a la China y a Estos Unidos en este resultado. Hay que destacar que en los últimos 35 años el país asiático ha experimentado crecimiento en su PBI por el orden del $10 \%$ como promedio y mientras que Estados Unidos lo hacía por el orden del 2,5\%.

Complementariamente a este indicador podemos encontrar en el Índice de Competitividad global como una variable que denota el avance de las principales economías del mundo. Si bien, los primeros que salen en el ranking no son los que poseen el liderazgo de la economía mundial, pero 
si son países que han logrado avanzar en los pilares básicos de competitividad que entre ellas está la educación, innovación e investigación (I\&D). Es más, como la población es pequeña en comparación con la China, EE.UU su renta per cápita supera al de las economías líderes del mundo Según un informe del Foro Económico Mundial, Suiza, Singapur, Estados Unidos, Holanda, Alemania entre otros tal como se aprecia en el cuadro No1 están en el top de las economías más competitivas del globo, si bien Finlandia ha bajados dos puntos con respecto al ránking 2015-2016, pero todavía sigue siendo un icono en donde la educación, la investigación, la tecnología se convirtieron en el soporte para sus desarrollo económico. China está en el puesto 28 en el ránking, el cual es un indicador que tiene que corregir una serie de políticas que fortalezcan su posición. Hay que tener en cuenta que lo económi-

Tabla 1.

Ránking de competitividad global

Fuente: Foro Económico Mundial / cdi.org.pe

\begin{tabular}{lcc}
\multicolumn{1}{c}{ PAÍS } & $\mathbf{2 0 1 6} \mathbf{- 2 0 1 7}$ & $\mathbf{2 0 1 5} \mathbf{- 2 0 1 6}$ \\
\hline Suiza & 1 & 1 \\
\hline Singapur & 2 & 2 \\
\hline Estados Unidos & 3 & 3 \\
\hline Holanda & 4 & 5 \\
\hline Alemania & 5 & 4 \\
\hline Suecia & 6 & 9 \\
\hline Reino Unido & 7 & 10 \\
\hline Japón & 8 & 6 \\
\hline Hong Kong & 9 & 7 \\
\hline Finlandia & 10 & 8 \\
\hline China & 28 & 28 \\
\hline Chile & 33 & 35 \\
\hline México & 51 & 57 \\
\hline Perú & 67 & 69 \\
\hline Bolivia & 121 & 117 \\
\hline
\end{tabular}

co es solo un pilar en su medición. El ránking de competitividad trabajado por el Foro Económico Mundial, proyecta señales como un país está avanzando en el campo de infraestructura, instituciones, entorno macroeconómico, salud y educación primaria, educación superior y capacitación, mercado laboral, eficiencia del mercado, entre otros. Si un país avanza simétricamente en todos estos pilares logrará posicionamientos importantes en la clasificación, tal como sucede con las Top 10 del mundo. Perú no sale bien ubicado el ránking, debido a una serie de debilidades estructurales, entre ellas la falta de una clara $y$ eficiente política educativa.

\section{La investigación en el mundo}

\section{En UNESCO (2013), se señala:}

En 2013 había aproximadamente en todo el mundo 7,8 millones

de personas empleadas a tiempo completo en actividades de investigación, lo que representa un crecimiento del $21 \%$ desde 2007 . Los investigadores suponen un $0,1 \%$ del total de la población mundial. Desde 2007, el porcentaje de personal investigador procedente de países con economías de ingresos bajos y medios aumentó un 5\%, llegando a alcanzar un $35,6 \%$ en 2013.

China, por sí sola, representa más de la mitad de este porcentaje; lo cual supone el 19,1\% de la fuerza laboral mundial empleada en actividades de investigación y equivale prácticamente al porcentaje que representa China en la población mundial: un 19,3\%.

En cuanto a la densidad de investigadores a nivel mundial, en el 2013 la media mundial de investigadores por millón de habitantes llego a 1,071 siendo de 1083 en el 2012. El país que más destaca en cuanto a este indicador es Israel que en el 2012 llego alcanzar 8.337 investigadores por millón de habitantes, dicho sea de paso fue la más alta del mundo, lo que supone más del doble de la densidad de EE.UU que fue de 3.984 en el 2012 y del Reino Unido 4.108 en 2013. Esta densidad era también muy elevada en 2013 en la República de Corea (6.553) y en Japón (5.195).

Estas cifras denotan que ningún país del orbe occidental y aquellos que poseen el liderazgo económico , financiero, Tecnológico, han dejado brechas de debilidad en la investigación, el hecho que avancen en cantidad de investigadores es la ruta perfecta para innovar y crear nuevos conocimientos o productos científicos inclusive en beneficio de la población mundial. Cosa muy distinta sucede en LAC, que están muy rezagados en materia 
de investigación, por el simple hecho de que esta actividad no es prioridad en las políticas del Estado. Este hecho se puede contrastar con la cifra de la UNESCO que señala que en América Latina y Asia Meridional -regiones que concentran el $8 \%$ y el $23,3 \%$ de la población mundial respectivamente- el porcentaje de investigadores respecto del total mundial es a grosso modo comparable: $3,6 \%$ en la primera y $3,1 \%$ en la segunda.

Toda esta diferencia en los resultados de la $\mathrm{I}+\mathrm{D}$, en parte es el reflejo del soporte financiero que se le asigna a esta actividad. Tal como se aprecia en el cuadro Tabla 2, los países y regiones más avanzados económicamente como son : EE.UU, Unión Europea y Japón y China como país emergente son los que muestran mayor monto asignado para financiar actividades científicas, producto de ello es su liderazgo en el mapa económico del mundo. En contraste con los países en vías de desarrollo como es Perú, Chile y Colombia en LA cuyo aporte no supera la barrera del $0.5 \%$ con excepción de Brasil que en 2013 llego al $1.24 \%$, por ello este país cuenta con la supremacía en la I+D en esta parte de la región.

\section{Educación en el Perú}

El Perú en estos últimos años, si bien es cierto ha avanzado en el tema educativo muestra de ello es el resultado de la última prueba Programa para la Evaluación Internacional de Estudiantes (PISA) que mide la capacidad lectora y matemática del educando, pero aún queda muchas anomalías de tipo estructural que se tiene que corregir si el reto es tener en los próximos años una plataforma educativa con competencias y capacidades para dar soporte a la visión económica y

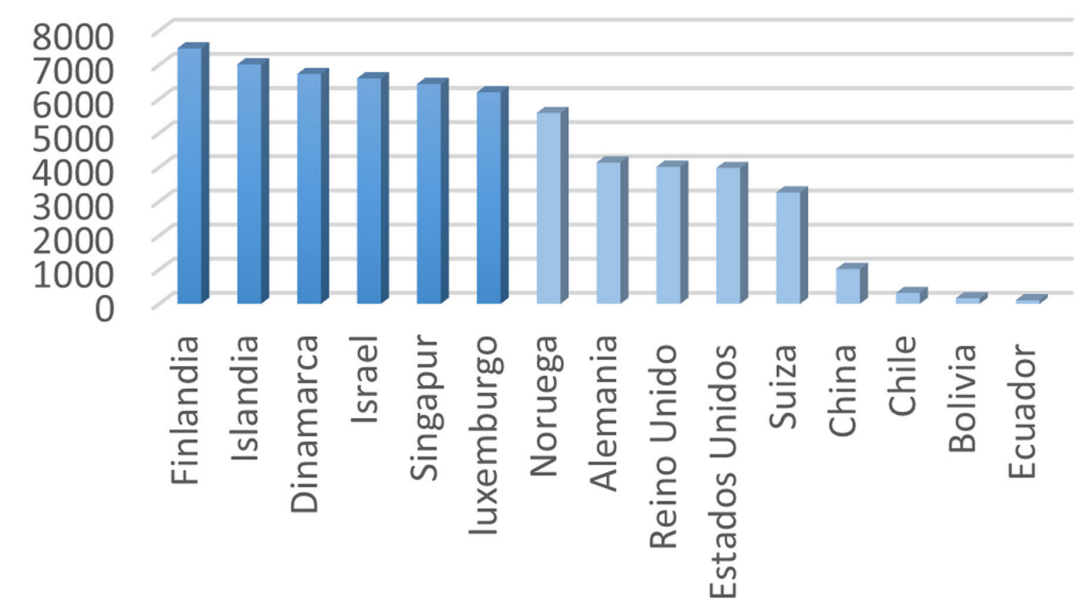

Figura 1. Investigadores dedicados a I\&D - 2012 - Por millón de habitantes Fuente: http://www.indexmundi.com/

Tabla 2.

Gasto en investigación y desarrollo por países - 2013 (\% PBI)

Fuente: Banco Mundial

\begin{tabular}{cc} 
PAÎ́S & \% del PBI \\
\hline EE.UU & $2,79 \%$ \\
\hline China & $1,98 \%$ \\
\hline Alemania & $2,99 \%$ \\
\hline Israel & $3,91 \%$ \\
\hline Finlandia & $3,55 \%$ \\
\hline Japón & $3,34 \%$ \\
\hline Reino Unido & $1,72 \%$ \\
\hline Singapur & $2,08 \%$ \\
\hline Corea & $4,36 \%$ \\
\hline Brasil & $1,24 \%$ \\
\hline Perú & $0,15 \%$ \\
\hline Chile & $0,39 \%$ \\
\hline Colombia & $0,26 \%$ \\
\hline
\end{tabular}

social del país. De lo que se haga ahora en el campo educativo, dependerá como el Perú se podría desenvolver en un futuro en los escenarios globales. Si se quiere competir con las economías desarrolladas, como Europa. Estados Unidos, Japón y emergentes como es a China, se tiene que invertir en educación de calidad e integral, lo cual implicaría fortalecer el componente infraestructura, capacitación y remuneración docente, apoyo eficiente de la gestión administrativa, es decir, todos los acervos tienen que avanzar simétricamente y sistemáticamente para obtener logros 
Tabla 3.

Resultado de la prueba PISA 2015

Fuente: PISA- OCDE

\begin{tabular}{lccc}
\multicolumn{1}{c}{ Países } & Ciencia & Lectura & Matemática \\
\hline Media OCDE & 493 & 493 & 490 \\
\hline (1) Singapur & 556 & 535 & 564 \\
\hline (2) Japón & 538 & 516 & 532 \\
\hline (3) Estonia & 534 & 519 & 520 \\
\hline (4) China & 532 & 497 & 542 \\
\hline (5) Finlandia & 531 & 526 & 511 \\
\hline (69) Perú & 397 & 398 & 387 \\
\hline (70) Líbano & 386 & 347 & 396 \\
\hline (71) Túnez & 386 & 361 & 367
\end{tabular}

tal como de observa por ejemplo en los países de la OCDE (Ministerio de Educación Cultura y Deporte, 2016). Es cierto que esta prueba (PISA) es un buen medidor para analizar la eficacia de las políticas educativas, pero la idea no es estar compitiendo en los últimos puesto como sucede con el Perú, sino a un largo plazo debe apuntar a lograr posiciones como Singapur, Japón, Estonia, China Taipéi, Finlandia, que a pesar de no ser una potencia económico con excepción de China, logran un desempeño impresionante en el tema educativo. Países que han focalizado a la educación en todos sus niveles como el

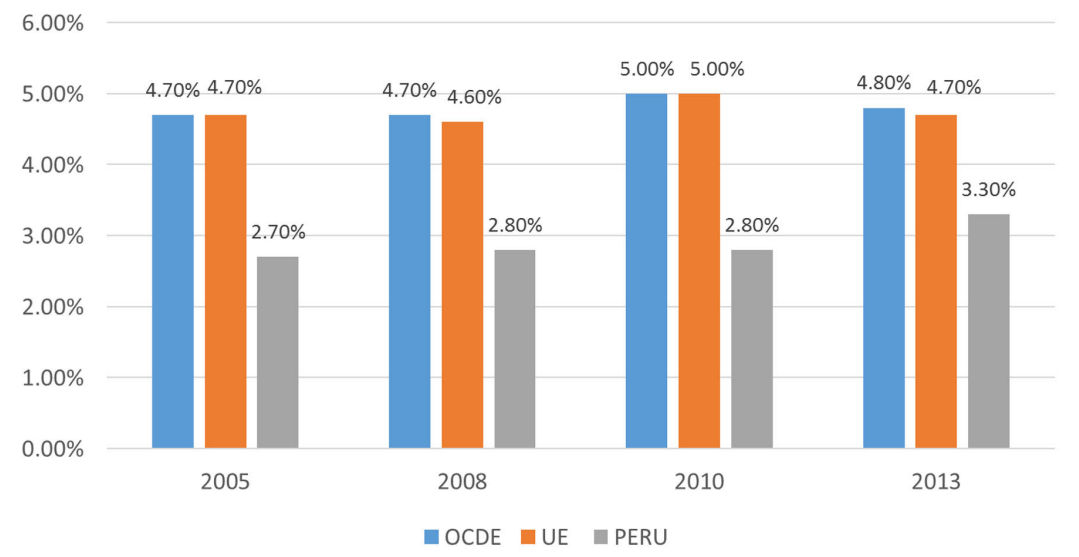

Figura 2. Gasto en educación con relación al PBI 2005 - 2013

Fuente: OCDE pilar básico para dar sostenibilidad a su bienestar económico y social.

Un hecho que hay que destacar que en este último resultado de evaluación educativa, es que el Perú ocupa el último lugar a nivel de Latinoaméporción más que todos los países de la región, pero las cifras de medición dejan muchas deficiencias que se tienen que corregir para seguir subiendo peldaños en la educación global. Es claro que los resultados antes mencionados solo es el reflejo de la ponderación que le dan los gobiernos a la educación en sus agendas de políticas rica, si bien se ha avanzado en pro- gubernamentales. Si la educación se conceptualiza como una actividad de primer orden estará en el primer lugar en las tareas gubernamentales. Bajo este marco cabe preguntar ¿Cuál ha sido el alcance presupuestario para posicionar el concepto de la calidad educativa en el Perú en comparación con las demás regiones del mundo?

Un dato relevante para evaluar el desempeño de la educación es la participación del Gasto con respecto al PBI, es decir el monto que se le asigna para que el sector financie sus partidas corrientes y de capital en correlación con la riqueza producida en un país. Es obvio pensar que si la tasa de participación del sector ya está pre establecida mediante una política gubernamental las oscilaciones presupuestales dependerá de cuan volátil sea el PBI, mejor dicho habrá una estrecha correlación entre las sensibilidades de los montos presupuestales y del comportamiento de este indicador macroeconómico. Bajo esta línea de análisis se muestra en la Figura 2 en donde se observa que los gastos en educación de los países de la OCDE y de la Unión Europea con respecto al PBI es casi el doble de lo que el Perú destina para financiar esta actividad, este hecho se ve reflejado en ciertos indicadores como por ejemplo, los gastos entre la OCDE y la UE van a la par, la asimetría se localiza con el Perú y que es común con algunos países de la región con excepción de Chile cuya participación del gasto con respecto al PBI llega en promedio al $4.7 \%$. Existe tendencia que en el caso del Perú en el 2021 se llegue a la meta del 5\% tan igual como se aprecia en los países miembros de la OCDE.

Otro indicador básico para evaluar el comportamiento del sector es el gasto educativo por estudiante a los tres niveles de estudio. Es decir, cuanto 
se destina en promedio a cada estudiante en los diferentes niveles de estudio. Se entiende que en el tiempo esta cifra debe ir mejorando en forma equilibrada y horizontal que denote igualdad de oportunidades para todos los estudiantes, lo cual significa que un alumno que se ubica en la zona rural debe tener el mismo monto asignado que el de la zona urbana, de esta forma se estaría cumpliendo con el principio de igualdad en la redistribución de la riqueza del país.

Según INEI el gasto promedio por estudiantes, de los tres niveles del 2007 al 2013 llego a $\$ 709.0$, pero si se toma en cuenta el gasto a nivel secundario el gasto al final del periodo del 2013, llegaba a $\$ 977.0$, resultado que se da por la asignación financiera a cada nivel de estudio, donde se aprecia menor participación del nivel primario. Estas cifras si los contrastamos con los gastos que llegan a nivel mundial que llega a $\$ 8000$ y en la región que asciende a $\$ 2500$, se puede apreciar las brechas financieras que juegan en contra del país y que se tiene que corregir si se quiere entrar en la OCDE que es la meta gubernamental. Estas cifras al final de cuentas se convierten en resultados académicos de los estudiantes. La evolución del gasto se aprecia en la Figura 3, caracterizándose por una tendencia creciente en el tiempo. Comportamiento que está altamente correlacionada con el desempeño económico, el cual es medido por el PBI.

La eficacia de la educación en su aporte al crecimiento económico, no solo depende de cuánto sean los recursos financieros que vayan destinados a potenciar el sector. Por ejemplo, el Estado podría asignar 5\% o $6 \%$ o $10 \%$ del PBI como lo hace Cuba para crear un sector moderno y competitivo, pero el hecho no solo es la cantidad de fondos, sino es como se utilicen

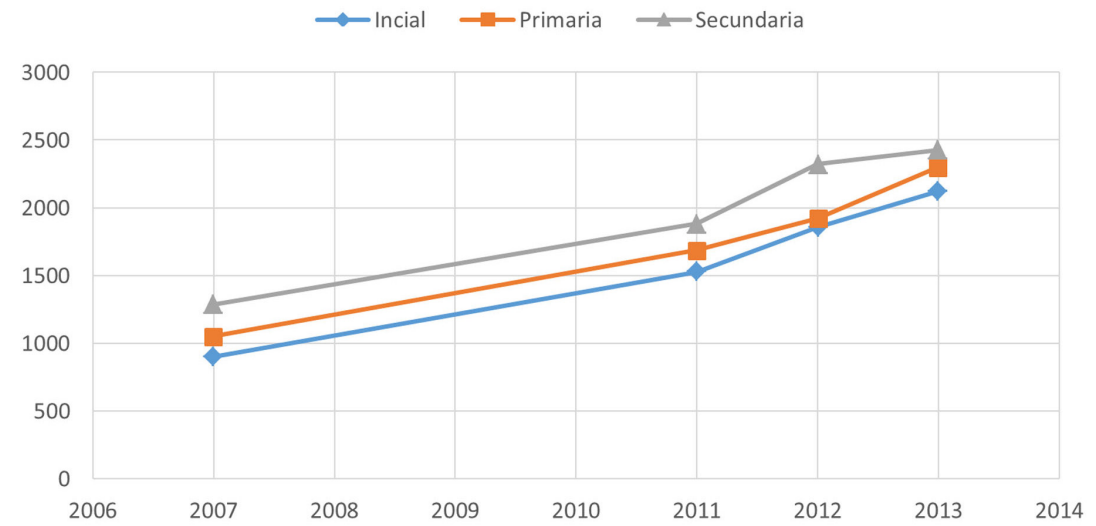

Figura 3. Gasto por estudiantes 2006 - 2013

Fuente: INEI

estos recursos, si se practica la optimización con la priorización y estos criterios se antepone las posturas políticas se podría conseguir buenos resultados, si esto sucede en los niveles básicos y se repite en el nivel universitario y postgrado, tal como sucede en los países del primer mundo, se estaría generando toda una plataforma de despegue para el desarrollo sostenido. La estructura curricular debe responder a las necesidades de las sociedad, los docente deben de tener la mejor calificación en términos de com-

petencias, las infraestructura debe brindar todas las facilidades para un óptimo aprendizaje y complementariamente debe de implementarse programas sociales donde el actor principal sea el educando. Si todas estas actividades son evaluado ex ante, ex post, se estaría abriendo una ruta para la calidad educativa, y con ello se formaría recursos con altos grados de competitividad. Todos listos para insertarse en el mudo laboral con las mejores competencias para crear riquezas con fines redistributivos.

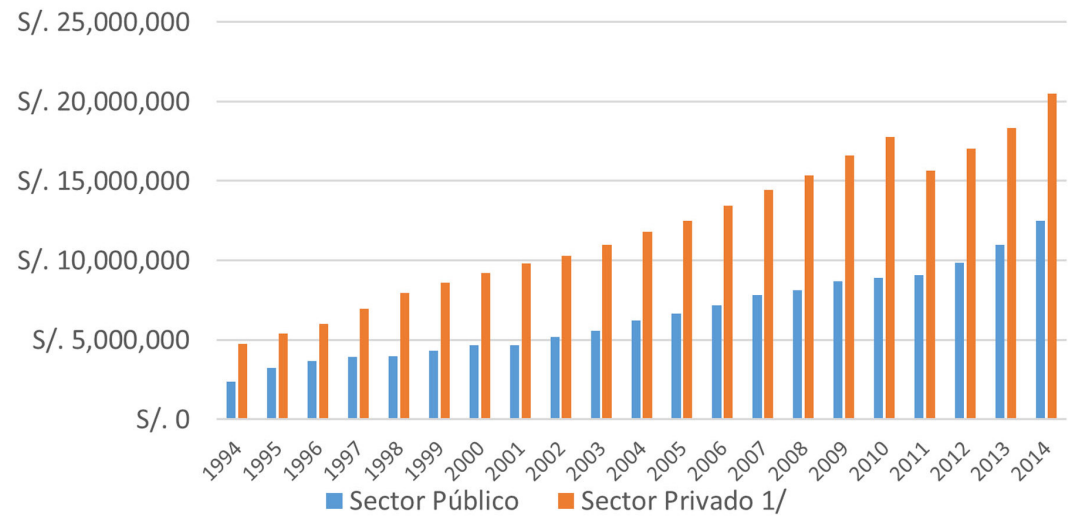

Figura 3. Gasto en educación 1994 - 2014

Fuente: INEI 
Gastos en investigación (I+D) en el Perú

Los estudios empíricos señalan que la inversión en $\mathrm{I}+\mathrm{D}$ el cual se va a materializar con innovaciones, patentes, nuevas tecnologías necesaria para mejorar las condiciones productivas de un país. Si en la agricultura se requiere mejorar el rendimiento por hectárea de las áreas de cultivo, es necesario hacer investigaciones que impliquen mejorar procesos, optimizar el uso de los recursos hídricos o recambiar el patrón genético de las semillas, para lograr todo esto se requiere laboratorios, apoyo financiero, infraestructura es decir es necesario montar toda una plataforma con la finalidad que el personal científico se sienta en óptimas condiciones de trabajo para desarrollar ciencia en este campo. El mismo caso se puede replicar en la minería, industria manufacturera, es decir en todas aquellas actividades económicas que producen riqueza (Marroquín y Ríos 2012).

El logro de este propósito no solo de ser preocupación del Estado como actor cooperante para desarrollar un batería de proyectos científicos,

Tabla 4.

Innovación en el mundo 2015

Fuente: WIPO - Elaboración propia

\begin{tabular}{lcc}
\multicolumn{1}{c}{ Países } & Puntos sobre 100 & Ránking \\
\hline Suiza & 68,30 & 1 \\
\hline Reino unido & 62,42 & 2 \\
\hline Suecia & 62,40 & 3 \\
\hline Holanda & 61,58 & 4 \\
\hline EE.UU & 60,10 & 5 \\
\hline Finlandia & 59,97 & 6 \\
\hline Singapur & 59,36 & 7 \\
\hline Irlanda & 59,13 & 8 \\
\hline México & 38,03 & 57 \\
\hline Perú & 34,87 & 71 \\
\hline
\end{tabular}

sino también deben de participar las empresas privadas por ser las más interesadas de que se desarrollen los productos derivados de la actividad científica. Como productos de las grandes transformaciones tecnológicas, en donde también hay espacio para las universidades, las empresas estarán en mejores condiciones para competir con mayores fortalezas en los mercados globales y no solo eso, el país como un todo contará con mayores competencias para ubicarse en un mejor lugar en el ránking de competitividad global. La inversión en ciencia y tecnología no es un desembolso que implique lograr un retorno en un corto plazo, pero si genera las bases necesarias para garantizar un crecimiento económico sostenido a largo plazo. Al contrastar el posicionamiento de estos países con su indicador macroeconómico y demás indicadores sociales se puede evidenciar una estricta correlación directa, lo cual amerita afirmar que este tipo de inversión si genera riqueza y bienestar para la sociedad.

A continuación, en la Tabla $\mathrm{N}^{\circ} 4$ se aprecia lo débil que es el Perú en el campo de la innovación. El país ocupa el puesto 71/141 alcanzado solo

34.87 puntos de 100. Esta cifra, que por cierto es alarmante, es el reflejo de la poca ponderación que se le da a la investigación como herramienta de desarrollo, y si se sigue bajo este mismo escenario el destino final solo será exportar concentrado de minerales y con ello estar anclados para siempre al mercado de commodities, lo cual no es ninguna ventaja en estos tiempos donde los mercados le dan mayor valor a los productos derivados de la ciencia o productos con elevado valor agregado.

En la Tabla 5, las cifras señalan dos cosas, primero, cuanto se investiga y segundo, cuanto se crece como país. La relación es muy simple, si un país no tiene una industria del conocimiento a gran escala simplemente estará condenada a ser considerada en vías de desarrollo, si por el contrario desarrolla toda una infraestructura para desarrollar las habilidades y competencias de las personas, tendrá una sociedad rica y con bienestar para todos. $\mathrm{El}$ más claro ejemplo de los efectos de no considerar a la investigación como un tema de primer nivel son las economías pobres africanas como Guinea, Togo, Sudan, que ocupan los últimos lugares en el ránking de innovación. Alcázar y Lozano (2009) consideran que:

La evaluación del impacto real de la ciencia y la tecnología debe ser considerada como un asunto central, con la finalidad de construir políticas científicas y tecnológicas acertadas, que produzcan innovación y que den solución a los principales problemas que caracterizan a los países en desarrollo. Los criterios hasta ahora utilizados para evaluar el mérito científico en los países en desarrollo, no son completamente adecuados para la toma de de- 
cisiones de políticas de investigación, al igual que no son adecuadas para otorgar financiación correctamente dirigida. (p. 124)

De todo el análisis se desprende de que la educación complementado con las actividades de investigación, desarrollo e innovación son actividades estratégicas para potenciar una economía a largo plazo y con ello mejorar los indicadores sociales, evidencia de ello son las economías desarrolladas, que en la industria del conocimiento han encontrado el verdadero motor de desarrollo sostenido.

\section{DISCUSIÓN}

La inversión en el capital humano en el Perú en los últimos años ha estado por debajo del promedio mundial y de la región, lo cual ha originado que los estándares en la calidad educativa y en las actividades de investigación no han podido corregir sus debilidades y por ello no han cumplido con el rol dinamizador de la economía, comportamiento macroeconómico que respondió más que todo a las inversiones privadas, a las bondades del mercado externo y las exportaciones sin valor agregado.

La inversión en ciencia y tecnología (I+D) en el Perú es uno de los más bajos a nivel mundial y regional, el

Tabla 5.

Gastos en I+D por regiones 2007-2013 - Porcentaje \% Fuente: UNESCO- Elaboración propia

\begin{tabular}{lcccc}
\multicolumn{1}{c}{ PAÎ́SES CON ECONOMÍAS } & $\mathbf{2 0 0 7}$ & $\mathbf{2 0 0 9}$ & $\mathbf{2 0 1 1}$ & $\mathbf{2 0 1 3}$ \\
\hline INGRESOS ALTOS & 79,7 & 75,6 & 72,6 & 69,2 \\
\hline $\begin{array}{l}\text { INGRESOS MEDIANOS } \\
\text { ALTOS }\end{array}$ & 16,1 & 19,9 & 22,7 & 25,8 \\
\hline $\begin{array}{l}\text { INGRESOS MEDIANOS } \\
\text { BAJOS }\end{array}$ & 22,5 & 24,8 & 26,6 & 28 \\
\hline BAJOS & 6,9 & 6,6 & 6,5 & 6,4 \\
\hline BAJOS INGRESOS & 1,2 & 1,2 & 1,3 & 1,3 \\
Total & $\mathbf{1 0 0 . 0 0}$ & $\mathbf{1 0 0 . 0 0}$ & $\mathbf{1 0 0 . 0 0}$ & $\mathbf{1 0 0 . 0 0}$
\end{tabular}

grado de participación con relación al PBI que solo llega al $0.15 \%$ se ha convertido en un factor, entre otros, que no ha permitido desarrollar proyectos de investigación de gran impacto, por ello la poca calificación que obtienen sus centros de investigación, incluyendo las universidades, en el ránking mundial. Los débiles logros que se obtienen en el campo de la $\mathrm{I}+\mathrm{D}$, reflejado en indicadores de innovación, patentes, competitividad no han coadyuvado a dar sostenibilidad al crecimiento económico. La poca inversión en desarrollo ciencia y tecnología en términos generales refleja la gran ocupación en sectores económicos tradicionales que no exigen ciencia ni mucho talento en el capital humano.

\section{REFERENCIAS BIBLIOGRÁFICAS}

Alcázar, E. \& Lozano, A. (2009). Desarrollo histórico de los indicadores de Ciencia y Tecnología, avances en América Latina y México. Revista Española de Documentación Científica. 32(3), 119-126. doi: 10.3989/ redc.2009.3.676

Dornbush, R., Fisher, S. \& Startz, R. (2015). Macroeconomía. (Décima segunda edición). México D.F.: McGraw Hall.

Gerald, A. (2007). Introducción a los modelos de crecimiento económico exógeno y endógeno. Edición electrónica gratuita. Texto completo http://www. eumed.net/libros-gratis/2007a/243/ indice.htm

Hanushek, E. A. \& Wößmann, L. (2010). Education and Economic Growth. En B. Peterson \& M. Gaw. (Eds.), International Encyclopedia of Education-Volumen2. Oxford: Elsevier.

Larrain, F. \& Sachs, J. (2002). Macroeconomía en una economía global. (2da ed.). Buenos Aires: Pearson Education. 
Marroquín, J. \& Ríos, H. (2012). Inversión en investigación y crecimiento económico: un análisis empírico desde la perspectiva de los modelos de $\mathrm{I}+\mathrm{D}$. Investigación Económica, 71 (282), pp.15-33.

Ministerio de Educación, Cultura y Deporte (2016). Panorama de la educación. Indicadores de la OCDE 2016. Recuperado de http://www.mecd.gob. es/inee/publicaciones/indicadores-
educativos/Indicadores-Internacionales/OCDE.html

Rodriguez, J. (2005). La nueva fase de desarrollo económico y social del capitalismo mundial. (Tesis para optar el grado de doctor en Economía). Universidad Nacional Autónoma de México, Ciudad de México, México.

UNESCO (2013). Situación educativa de América Latina y el Caribe: hacia la educación de calidad para todos al 2015. Recuperado de http:// repositorio.minedu.gob.pe/handle/123456789/3650

UNESCO (2015). Informe de la UNESCO sobre la ciencia: hacia 2030. Resumen. Recuperado de http:// repositorio.minedu.gob.pe/handle/123456789/4850 